\title{
Statistical inference on lattices
}

\section{David K. Pickard}

In many scientific disciplines, there is frequently a need to describe purely spatial interactions among objects located at the sites of a lattice. Of particular interest are the equilibrium states of physical and biological phenomena occurring simultaneously at sites in more than one dimension (for example, ferromagnetism, crystal formation, patterns of infection). Markov random fieids form a wide class of intuitively appealing models for spatial interaction. Binary ones have been studied extensively in statistical mechanics where they are known as Ising models.

In the biological and ecological context, it is the fitting of data and analysis of given patterns which is most relevant. Statistical inference for Markov random fields is a relatively new field. In particular, Ising models are notoriously difficult to analyse and the absence of constructive characterizations has rendered Monte Carlo simulation an ineffective tool for comparing various statistical techniques.

This thesis provides a basis for asymptotic maximum likelihood inference for binary Markov random fields. The general method is illustrated by the detailed analysis of the classical Ising lattice with nearest neighbour interactions. However, the resulting limit theorems for this model are of considerable interest in their own right. Some rather surprising technical problems arise in connection with the application of the limit theorems. A solution is presented.

Also, a class of relatively tractable Ising models, which admit constructive characteriżations and direct simulation, is introduced in this thesis. The properties of these models are explored. In particular, they

Received 8 June 1977. Thesis submitted to the Australian National University, June 1977. Degree approved, August 1977. Supervisor: Professor P.A.P. Moran. 
generally have a geometric correlation structure and contain embedded Markov chains. Moreover, they can further be used in simulation studies to compare the relative effectiveness of various methods of spatial analysis.

Finally, although all the results are for binary models, the tools are useful in much wider generality. They apply, for example, to gaussian Markov random fields as well. 